Under certain pathologic conditions, this equilibrium is more easily disturbed. I shall only refer to the increased possibility of producing reactions in case of diminished functioning of the excretory organs. It is a common practice to test kidney function before and during a course of arsphenamin or its substitutes, for obvious reasons.

In special cases, previous treatment with a drug which produces a cumulative effect has also to be taken into account. In a case of malignant malaria which is treated intensively with quinin by mouth, it is decidedly dangerous to give the intravenous dose of quinin ordinarily recommended for this purpose without first allowing the patient to dispose of the drug previously given; and this is equally true of the arsenical treatment of syphilis, and of the intravenous use of strophanthin in cases which are under treatment with digitalis by mouth.

Having seen that intravenous medication is not necessarily superior therapeutically to other modes of drug administration, and having pointed out the numerous possibilities of injury to the patient inherent in this method, we should strive to simplify the technic of the administration of drugs wherever possible, in order to avoid the necessity of the injection of the medicament directly into the circulation. The subcutaneous injection of morphin and similar drugs is an exceedingly simple procedure, which very often is left to the attending nurse. Would it not be a great step forward if such drugs as arsphenamin or its substitutes could be given subcutaneously?

During the last year, in collaboration with Miss Dyer, Dr. Johnson and Mr. Thompson, I have studied such a preparation experimentally, and so far it meets all the essential requirements. It is prepared from arsphenamin, formaldehyd and sodium bisulphite; its aqueous solutions are very stable and do not increase in toxicity on standing for twenty-four hours or on vigorous shaking. Its parasiticidal effect is extremely constant, and it does not cause any local reactions if injected hypodermically. The chemotherapeutic index or, in other words, the ratio between the curative and the maximum tolerated dose is as good as in the case of arsphenamin. The drug is now being tested clinically, and, should our hopes be fulfilled, I believe that it will have a considerable effect on the practical problem of the control of syphilis; for it stands to reason that such a preparation would make it possible for physicians who have not the required experience with intravenous arsphenamin treatment to give the treatment in this simple manner, if necessary under the general supervision of an experienced syphilologist, as advocated recently by Stokes.

\section{DISEASE CONTROL AND PREVENTION}

I believe that it should be emphasized in this connection that intravenous medication, of necessity, places decided restrictions on the treatment as well as on the prevention of certain diseases, on account of the more or less difficult technic inherent in this method. Take, for instance, the problem of the control and eradication of syphilis; it surely stands to reason that a simple technic of treatment, such as the subcutaneous injection, would make it possible to put a larger number of syphilitics under the controlling influence of the arsenicals; and this would unquestionably help in checking the spread of this disease, and it might also assist in its eradication.
Again, the practicability of the prevention of malaria by means of the oral administration of quinin rests exclusively on the fact that it is possible to produce a prophylactic effect by taking quinin tablets by mouth; and I doubt whether anybody would claim that the prevention of this disease could be made a feasible proposition if quinin had to be injected intravenously for this purpose. Therefore, every effort should be made to render the administration of such drugs as simple and safe as possible, without, however, sacrificing their therapeutic efficiency. Intravenous medication, to my mind, will never serve this purpose, and will always have a more or less restricted field of usefulness.

\section{THE REAL THINGS IN MEDICINE*}

$$
\begin{aligned}
& \text { HUBERT A. ROYSTER, A.B., M.D. } \\
& \text { RALEIGH, N. c. }
\end{aligned}
$$

The minimum requirements for a career in medicine are a preliminary education, four years in a medical school, and a license to practice. While these requirements are indispensable, they are by no means sufficient. Three higher attributes stand out as real elements that make for a successful physician; without these, real success in medicine is impossible : brains, culture and character.

\section{BRAINS}

Brains are the alpha and omega of the man of medicine. We all recall the artist who, when asked what he mixed his paints with, replied: "With brains, sir." Not long ago when I was on the way to talk to college students on medicine as a profession, the wife of one of the faculty said: "Tell them that the one thing they ought to have is brains." She had made my speech. To begin the study of medicine without two good cerebral hemispheres thickly covered with a deep cortical layer is to invite failure. No encouragement should be extended to those about to enter the profession of medicine or to those already in to continue, unless they are supplied abundantly with gray matter. The misfits in medicine, as well as in other occupations, are due largely to a lack of understanding. There are some in the cornfield who ought to be in medicine; there are some in medicine who ought to be in the cornfield.

The study of medicine bestows no more brains and adds not one cubit to the mental stature. It provides only a few more implements of the mind to be used for good or for ill. Unless the roots are deeply planted in the soil of real learning, the study of any science narrowly pursued takes away from the natural resources of the mind more than it puts in. In other words, a finely endowed intellect is needed to carry on in the realm of science, to withstand its temptations toward the illogical, to keep clear headed in the midst of fact and fancy.

Such endowment comes not from science itself; it issues permanently from a mind nurtured in the ways of thinking. The handmaiden of brains, essential for the physician, is common sense, which is simply the ability to put brains to good account. In the words of Holmes, "Science is a first-rate piece of furniture for a man's upper chamber if he has common sense on the ground floor. But if a man hasn't got plenty' of good common sense, the more science he has the worse for his patient."

* President's address, read before the Medical Society of the State of North Carolina, Winston-Salem, April 25, 1922. 


\section{CULTURE}

Make no mistake about culture. Culture is refinement, accuracy, poise, resourcefulness; it is not effeminacy, weakness, conventionalism, impracticability. We have been getting too far away from the humanities, from classical education, from academic scholarship, if you please. In the quest of science-and there is no nobler pursuit-we have set up utilitarian courses, called premedical and certainly premeditated, for the purpose of reducing to a minimum all those things which do not bear directly on the matter in hand, and swelling to a maximum those that are concerned in the material things of medicine. In this we have left out the very bed rocks of learning: the capacity to interpret the phenomena and the power to express the findings. If the average trained laboratory worker in our country today has any weakness, it is his inability to convey his ideas, to put down what he has done, to express his results in terms clear and terse. Generally his work surpasses his words. And it is not the misfortune of the individual, but of the plan which essays to substitute scientific research for sound scholarship; to get the one without the other, when we may have both. It is the common failing of the later generation of medical men that they do not write so lucidly or think so accurately as those just before them. There are, of course, numerous and notable exceptions. But the observation is probably correct, and its explanation lies in the small stress placed on actual scholarship required of one entering the profession of medicine.

We are living in an age of inaccuracy. We are inaccurate in thought, in speech, in spelling, in writing. We know a great deal ; but do we know anything very well? Short cuts and practical preparation are the order of the day. Language, the only medium through which thoughts are given out, has been almost forgotten. Will it be considered very old fashioned if $\mathrm{I}$ should suggest that the neglect of the languages, and particularly the banishment of Greek, may be responsible for our loose thinking and our lack of scholarship? The value of Greek for the medical student might be a theme for a discourse in itself. If you should go over the evidence, you would be surprised to find how much medicine owes to the Greek language, what a very large number of our words referring to diseases, operations and organs are derived from the Greek-fully as many as from Latin. And many of those coming through the Latin were taken originally from Greek. "We suspect, too, that our men of science who are supposed to be opposed to 'so much Greek' must study that language secretly. or they assuredly could not name the tools of their own trade." 1 The chief advantage of the study of Greek is a training in accuracy, in the expression of nice shades of meaning, the very essence of a cultivated mind engaged in scientific thought. We cannot divorce science and culture; we cannot go on rearing a race of seekers after truth who are not trained thinkers; we cannot fail to perceive that the education of a candidate for a learned profession means for us, as it has meant for all the older nations, a thorough grounding in the ancient and honorable arts and classics before we approached the special study needed for our dignified calling. That way trod our great fathers, who outstripped us with the means at their disposal; that way lies our hope of elevation, of bringing back the well rounded medical man and adding to him the marvelous scientific attainments of the present age. My thought

1. London Saturday Review, Aug. 16, 1913. was expressed by Thomas L. Stedman, ${ }^{2}$ in these words : "Some day the pendulum will swing the other way and a new renaissance will once more join culture to knowledge to make the perfect physician."

\section{CHARACTER}

Detracting not one whit from the appeal for better mental equipment, it appears even more timely to emphasize the greater need for moral qualification in the physician. By this no reference is meant to righteousness or to worlds to come, to religion or to denominationalism, but only to the possession of a sound moral sense. The character of a physician is his most priceless property. It is what he stands for and whom he stands with; it is his attitude toward life; it is "what God sees him do in the dark." Truth is the goal ; it develops character, and character tells in one's work. Too many are entering the medical profession without good working consciences, without a keen appreciation of the difference between right and wrong ; too many are coming into an honored and honorable calling with low standards of trade and traffic in their souls; too many get in who are not gentlemen to begin with and cannot be made gentlemen to end with. Today the young physician too frequently feels compelled to ask his associates, "What do I get out of it?" when he might better ask himself, "What am I putting into it?" Greatly to be desired is a stronger effort to train the moral perception of medical students. They must be inspired toward a high-minded estate. In the speech and bearing of graduates of certain medical schools can be recognized just exactly the outlook on life and the regard for our profession held by their teachers. It is an unconscious percolation. The fine sense of intuitive right-thinking and truth-loving can shine through both precept and example. Morals cannot be legislated into people ; but ideals can be educated into them.

\section{PREPARATION FOR THE PRACTICE OF MEDICINE}

My tribute to science is the greater because of $m y$ contention for broader preparation before embarking in its service. I heartily join with Moses Harvey in saying that "science is teaching man to know and reverence the truth and to believe that only so far as he knows and loves it can he live worthily on earth, and vindicate the dignity of his spirit." Medicine is both a science and an art; it has to do both with knowing and with doing. It is not an exact science-more's the credit, for exact sciences are inert and uninteresting. But the one big asset for the medical man is the acquisition of the scientific attitude in his student days. Every physician should remain a student the whole of his natural life. A pitiable sight is the practitioner who was known as a "good student" but who, after five years more or less, has degenerated into a routinist, never reviewing his former knowledge or adding to his original store. Such a man, though perhaps skilled in so-called practical affairs, forgets more and more medicine each year, until finally it is difficult for him to keep up. He begins to decry theory and to extol practice; he thinks only of results and never of causes; he speaks magnificently of "common sense" and "letting Nature take its course," but rarely uses the one or permits the other.

The trouble with this type of person is that he has never acquired the scientific spirit. He fell short in the

2. Stedman, T. L.: A Practical Medical Dictionary, New York, William Wood \& Co., vii. 
early approach and missed the mental hole. The eager student loves learning for its own sake and believes that no knowledge is useless, if it is correlated. He understands that science is organized knowledge. The scientific attitude means that nothing must be accepted unless it is known; that nothing can be known until it is approved ; that everything shall be met in the spirit of hesitant belief. This smacks of pessimism, and one poet at least has been led to exclaim:

\section{$O$, star-eyed science! Hast thou wandered there,}

To waft us home the message of despair?

But nothing could be more unjust. It is not despairing to search for the truth. The challenge is, "How much do you know?" and "How can you prove it?" The doubting Thomas has a place here.

What has no place in science is the post hoc, ergo propter hoc habit of mind. It is the most dangerous of all doctrines for the medical man. It is the rankest empiricism and furnishes the platform for all quacks and impostors. "Whereas I was blind, now I see" is an insidious appeal to effects in utter disregard of the means-the cry of the charlatan. Montaigne wrote that when a man is sick and gets well, he cannot say whether it was because of the remedies used, the lapse of time, or his grandmother's prayers. It requires a powerful mental effort to get away from the belief that whatever happens after an event is always on account of it; but we must get away from this belief or we shall not retain our clear thinking.

After all, it is the acquisition of knowledge which is enticing and inspiring, not merely the possession of it. "If I held truth captive in my hand," says Malebranche, "I would let it fly that I might again pursue and capture it." And it is well to remember that not all the new is scientific, nor all the old empiric. Science teaches us to unlearn many things each day and to relearn as many the next; for there is scarcely anything new except what has been forgotten.

\section{GROUP' PRACTICE}

A turn in the affairs of medicine is just before us. Whether that turn will be toward new and untried fields or along some old and well-worn paths, the future - the near future-will determine. The struggle is on between medicine as practiced by the private physician in the time-honored relation, and the practice by groups in a collective fashion. No matter what may be the arguments on the one side or on the other, the situation will work itself out precisely in accordance with the desires of the public, the results of methods in use and the attitude of physicians.

Owing to the multiplicity of technical detail and the accumulating mass of new discoveries in-modern science, it is now more than ever true that no man diagnoseth to himself. It was never true that any one man, looking at the patient through the peephole of his slender specialty, could compass a complete diagnosis, or that a cursory inspection in the old way by the ordinary practitioner would round out a survey of the case. But always there has been a demand for the man who could find out everything there was to know about his patient, whether or not he himself could carry out all the detailed investigations and therapeutic indications. The main reason for requiring assistance in the diagnosis of disease is the need for particular apparatus, in the use of which some are skilled, to elucidate findings not brought out by the general physical examination.
Thus grew up specialism and thus were developed instruments of precision. But there never has been, and never will be, anything to take the place of cultivated brains and trained special senses. This duel instrument, ready for instant and proper use, will be found as the principal equipment in the office of the real physician. Hear a layman's view : ${ }^{3}$

The real pest among reputable physicians is the young man who expects his patients to pay for his needlessly high overhead expenses. He may be known by his spacious and elaborate offices and waiting rooms, buttoned door boys, sleek secretaries, fluttering office nurses and powder monkeys of both sexes, and an all pervading shimmer of white enamel, mechanical novelties and glittering metal work. Not infrequently the young practitioner who indulges in all these fripperies is trying to put over a poor piece by costly stage effects. He sometimes forgets, and his patients still oftener fail to realize, that what he really has for sale resides in his own cranium, and that mere style, atmosphere and scenery are poor substitutes for knowledge, experience and technical proficiency.

Unguided by intellect, instruments of precision become instruments of confusion, for they are not infallible and are wofully subject to the human equation. Rightly used and rigidly interpreted, they mean much in proving a diagnosis; regarded as the only means in arriving at conclusions or depended on as unfailing agents, they are but disappointments. Great pity should be felt for the inexperienced beginner who expects his microscope, his hemocytometer, his cystoscope, his chemical reagents and his metabolimeter to solve all the mysteries of diagnosis and to spell accurately the name of the disease. If I should see any such, I would say: "Let us first take a look at the patient; use our minds in obtaining his story; perform a laying-on of hands; after which we will ask for the aid of scientific apparatus which may either confirm or reject our observations. And if the laboratory findings do not agree with the clinical signs, then so much the worse for the laboratory."

And what will become of our specialism? Where will it end? Will there be need for its regulation and control? Undoubtedly the existence of specialties is the proof of their necessity, and their development has been the crowning achievement of modern medicine. But increasing subdivisions of specialism give us pause, so much so that by reaction we are beholding a newer specialist - the general practitioner. $\mathrm{He}$ is in a class by himself, left in charge of those who are actually sick, with the issues of life and death on his hands, both early and late. He is the keystone of the medical arch; without his support the whole fabric would fall; family after family are depending on him for counsel, relying on his judgment in every crisis. Of what use are specialists unless there is the intermediary to say when they are needed? And since the general practitioner has by distinction become a specialist and expected to know a little about everything, why should not the professed specialist be required to know something of general medicine? One finds oneself somewhat in agreement with the recently suggested proposal that examining boards may require five years in general practice before allowing the privilege of going into a specialty. How else may be curbed the tendency to divide the body into compartments for seclusive attention without a working knowledge of the whole system? These questions also will furnish their own answers.

3. Saturday Evening Post. 
Many are rushing into group medicine as a solution of the whole matter. Such practice brings to bear upon each case the combined study of a number of specialists, who, examining the patient individually, finally come together for establishing a complete diagnosis, or leave it to one member of the group to pronounce the judgment. Work in groups has come to stay; for it is inevitable that men will associate for mutual help and encouragement. Team work is the modern slogan, and it is the way to win the game. But if the group discounts the individual and the individual forgets the patient, we have, not group medicine, but gang medicine; and their product is syndicated science, than which nothing could be more unscientific. One very notable group clinic and several others of lesser fame and more recent date have served to popularize the combined method of practice, and diagnostic groups are springing up all over the land. There are some with only two men in them (reminding us of the close corporations in which one partner is the president and treasurer, and the other, vice president and secretary), and others containing scores of men; there is the peripatetic clinic, in which taxicab fare is a considerable item; there are those conducted under one roof either of a separate institution or on one floor in an office building; there is the community hospital with its broad choice of consultants and naturally a subgrouping among these. While there is much to be said in favor of any form of associated effort, certain fallacies and dangers lurk in group practice. Primarily it is well to recall that "a chain is no stronger than its weakest link," and unless the men on the team are of equal capacity in their several branches, the resultant report will be onesided. There is danger, too, of everybody's business being nobody's business, so that the diagnosis produced may be a compound of the average forces of a factory, ground out piecemeal. An impersonal mark is likely to attach to all such methods to rob the process of any human touch and to afford the greater chance of error, especially if the interpreter for the group sees the patient only for a short sitting on the last day of the ordeal. Of necessity, the head of an important group is compelled to be a genius in his sphere, and all groups which have succeeded scientifically have had such heads.

Free and frequent discussion has waged over the group question and its allied problems. My feeling is that the group idea is one of the most useful modes of service in medicine. But no group should be iron clad or unwieldy; its tendency to send out machinemade work should be changed into that of a handpicked product; it should stop straining at gnats and swallowing camels, and learn that the group was made for the patient, not the patient for the group. Just as strongly should we realize that the practice of medicine will never-and should never-get away from the personal relation of patient and physician, and that always we shall depend for our health and our happiness on the daily visits of the physician from house to house,

Battling with custom, prejudice, disease

As once the son of Zeus with death and hell.

\section{ADJUSTMENT TO CHANGING CONDITIONS}

A spirit of uncertainty pervades our rank and file. The existence of a subconscious fear that something or somebody will take away pristine rights, privileges and emoluments cannot be doubted. Nor can it be denied that this fear is natural and justified by appear- ances. What with welfare boards, full-time instructors in health, community service, vital statistics laws, narcotic restrictions, prenatal clinics and the like, physicians have begun to feel that they are "preached to" overmuch, that they are "regulated" beyond endurance, and that their confines are being invaded. Such expressions are heard in different quarters from time to time. Much as we may sympathize with them, the truth is that the fear which they denote is unfounded. We shall not lose anything unless we divest ourselves of it; we shall not have anything taken away from us, unless we prove unworthy to keep it; we shall not fail to receive any reward unless we sidestep the labor and the waiting necessary to secure it. The only harm that can come to us is the harm we may do ourselves by sins of omission and commission. The upsetting questions most of us are asking ourselves are: What are we going to do about it? where will the encroachments cease? how shall we adjust ourselves to the inevitable conditions? I will tell you what we are going to do: We are going to change, to progress. We shall go forward; we will meet and master every disturbing difficulty.

I have appealed to the fellows of the various component societies to keep in mind the county as the unit of organization and to remember that, for our common interest, attendance is the keynote, and cooperation the watchword; I emphasized the importance of the district societies and approved of plans to conduct their meetings according to a definite program, regarding them as miniature state societies; I sought to dignify the position of councilor, to exalt the duties of this officer, representing as he does in his district the power and influence of the parent organization. Finally, each member was urged to feel that the whole structure of organized medicine in state and nation hinged upon his own local status.

\section{CONCLUSION}

I have an ideal to express for this society. First, I would have it conform in every particular to the specifications set forth in Article II of the constitution. adopted in 1903, which stated that "the purposes of this society shall be to federate and bring into one compact organization the entire medical profession of the state . . . with a view to the extension of medical knowledge and to the advancement of medical science; to the elevation of the standard of medical education and to the enactment of just medical laws; to the promotion of friendly intercourse among physicians, and to the guarding and fostering of their material interests; and to the enlightenment and direction of public opinion in regard to the great problems of state medicine; so that the profession shall become more capable and honorable within itself, and more useful to the public in the prevention and cure of disease, and in prolonging and adding comfort to life." I would have this society embody these principles in the conscience of its every member, especially recalling the significance of the order in which the principles are presented; I would have it a place where righteousness reigns and no mean thing may live; I would make it a guild and not a trade union; I would keep it a haven where politics doth not enter and where selfish exploitation doth not corrupt; I would wish every one to think of not what the society is to give him, but what he is to give the society; I would want it a communion where freedom rules, where each fellow may have the aspiration and the opportunity to develop what is in him; but I would also carry its standard so high that we may 
draw all men up to it and never for one moment trail its colors in the wretched dust of the unqualified and the unfit.

We have every reason to feel encouraged. My note is one of optimism. If only we may glorify brains as the consummate need; if we reverence culture as a means of grace; if we set character above circumstance, then indeed shall we grow in capacity, refinement and virtue, assuming our rightful stations as devotees of a learned profession and champions of the real things in medicine. We cannot fail, we cannot lag behind, if we serve these purposes. May we not all stamp deep down in our minds and hearts the cheering message of our first president, the immortal Strudwick, who, seventytwo years ago, gave voice to these lofty words:

Neither the apathy of friends, the cold neglect and deep injustice of legislation, nor pampered quackery and empiricism can stay its onward course. True medical science will, like the majestic oak, withstand the shock and storm of every opposition. It has been beautifully compared to a star, whose light, though now and then obscured by a passing cloud, will shine on forever and ever in the firmament of Heaven.

\section{SIGNIFICANCE OF THE EXTRINSIC MUSCULATURE OF THE LARYNX}

ITS RELATIONSHIP TO CERTAIN DISORDFRS OF TIIE VOICE *

\section{ELMER L. KENYON, A.B., M.D. CHICAGO}

During the twenty-four centuries of historical knowlelge of the developments in laryngeal physiology, long periods, even of centuries, have elapsed during which no advancement in knowledge of the laryngeal function is known to have been recorded. And it may not be without a useful purpose to note here that evidence is: not wanting to cause one to wonder whether the laryngologist may not now be passing through one of these listless, or self-satisfied, periods with respect to laryngeal physiology. An examination of numerous recent laryngeal textbooks and general physiologies seems to warrant such a fear. One turns over the accounts of the physiology of the larynx in textbook after textbook without encountering any reference at all to physiologic knowledge of the larynx of the first importance, knowledge already nearly or quite a half century old. Such neglect of the very bases of laryngeal knowledge can but serve to retard progress in certain phases of laryngeal therapy.

If we are to understand the function of the larynx, it is first necessary to understand what the larynx is, both anatomically and physiologically. The anatomic definition is arbitrary and limits the meaning of the term to the laryngeal box itself, including the epiglottis. But a physiologic definition admits of no such arbitrary limitation. The physiologic larynx must be defined as the entire physiologic mechanism on which the movements of the vocal cords depend.

The laryngeal box is not only a suspended organ, movable upward and downward (through a range of about $3 \mathrm{~cm}$.) and in all other directions, excepting directly forward, to an important though limited

* Read before the Section on Laryngology, Otology and Rhinology at the Seventy-Third Annual Session of the American Medical Associa. tion, St. Louis, May, 1922. degree ; but it is movable only with, and as a part of, the hyoid bone, and the base of the tongue, and even of the lower jaw and floor of the mouth. Particularly, the larynx, hyoid and base of the tongue cannot but be considered as a physiologic entity. The larynx is suspended from the hyoid bone, the hyoid from the base of the tongue, the tongue from the lower jaw and the skull. All three are intimately involved in a complicated musculature whose action alters their relationship to each other and to the fixed bony structure of sternum, spinal column and skull. The component parts of this musculature are so numerous and the musculature is so complicated that a complete understanding of the combined movements requires much more investigation than has been undertaken up to this time. But one fact is not hard to make out; namely, that all parts move together and in harmony to produce even the simplest attempt at phonation by the vocal cords. They constitute definite factors in vocal cord physiology and all must be considered as belonging to the physiologic larynx.

The first definite statement which I have found in the literature, conveying the thought that extrinsic muscular action is always an accompaniment of vocalization, is that made by John Wyllie, ${ }^{1}$ in 1886 . In discussing the mechanism of pitch production, he says, "The position of the larynx varies with every note."

Reliable studies of the relationship of the extrinsic muscular action to the movements of vocal cords were made by Brücke, ${ }^{2}$ in 1856 , von Meyer, ${ }^{3}$ in 1884 , and John Howard, ${ }^{4}$ in 1886 , as well as by others.

Nature approaches the problem of vocal cord action in no partial or haphazard spirit. She approaches it rather with all the needs of vocal and musical physiology well in mind. The demonstration of this truth lies in the fact that no central nervous plan of laryngeal action has been provided, excepting that which compels the coordinating action of all the complex musculature of the larynx, both intrinsic and extrinsic. We, as human beings, are not afforded a nervous control of the intrinsic muscular mechanism alone. The nervous centers of vocalization which we may employ are only those which control the fifty or sixty distinct muscles, intrinsic and extrinsic, all of which must function for the simplest vocal act. ${ }^{5}$

The fact that combined action of the intrinsic and extrinsic muscular systems of the larynx is necessary for vocalization is further demonstrated by their instinctive united employment in producing the laryngeally produced crying sounds of the babe as he enters the world.

Only by harmonizing these two great physiologic facts, the production of voice by the adjustment and vibration of the vocal cords, immediately due to the action of the intrinsic muscles, and the constant coincident extrinsic movements of the laryngeal musculature, is it possible to understand the physiology of the larynx.

The immediate basis of vocalization lies in the manner of adjustment of the vocal cords to each other. Definite adjustments of the cords for pitch production

1. Wyllie, John: Disorders of Speech, 1886, p. 475.

2. Brücke: Grundzüge der Physiologie und Systematik der Sprachlaute, Vienna, 1856

3. Von Meyer, G. H.: The Organs of Speech, New York, 1884 4. Howard, John: The Physiologic Basis of Artistic Singing, Boston 1886.

5. The motor centers for the speech function control, at the same time, the function of chest contraction and expansion. If the muscles of the chest were included in the foregoing estimate, it would be found that the action of approximately 100 muscles was required for the production of any manner of vocalization. 